\title{
Utilização do software IRAMUTEQ em pesquisa de abordagem qualitativa: relato de experiência
}

Use of IRAMUTEQ software in qualitative research: an experience report

Uso del software IRAMUTEQ en investigación cualitativa: informe de experiencia

\author{
Fernanda Garcia Bezerra Góes ${ }^{\mathrm{I}}$, Andressa Silva Torres dos Santos ${ }^{\mathrm{II}}$, Brenda Lucas Campos ${ }^{\mathrm{III}}$ \\ Aline Cerqueira Santos Santana da Silva ${ }^{\mathrm{IV}}$, Liliane Faria da Silvav, \\ Luiz Carlos Moraes França ${ }^{\mathrm{VI}}$
}

\begin{abstract}
Resumo: Objetivo: relatar a experiência de utilização do software Interface de $R$ pour les Analyses Multidimensionnelles de Textes et de Questionnaires (IRAMUTEQ) como ferramenta informatizada de apoio à análise de dados textuais em uma pesquisa qualitativa em saúde. Método: tratou-se do relato de experiência acontecida entre março e junho de 2019, a partir de uma pesquisa qualitativa desenvolvida por meio do Método Criativo-Sensível e com o uso do IRAMUTEQ. Participaram puérperas e familiares de recém-nascidos de uma instituição hospitalar pública localizada na cidade de Rio das Ostras no Estado do Rio de Janeiro, Brasil. Resultados: foi possível constatar que as diferentes técnicas de análise lexical disponíveis no IRAMUTEQ permitiram discriminar, claramente, os conteúdos semânticos referentes às pretensões dos cuidadores sobre os cuidados a serem desempenhados com o recém-nascido em casa. Conclusão: essa ferramenta informatizada mostrou-se útil, adequada e fidedigna, qualificando o processo de análise textual.
\end{abstract}

Descritores: Pesquisa; Pesquisa em enfermagem; Pesquisa qualitativa; Metodologia; Software

\begin{abstract}
Objective: to report the experience of using the software Interface de $R$ pour les Analyses Multidimensionnelles de Textes et de Questionnaires (IRAMUTEQ) as a computerized tool to support the analysis of textual data in qualitative research in health. Method: this was an experience report that took place between March and June 2019, based on qualitative research developed through the Creative-Sensitive Method and using IRAMUTEQ. Newborns and family members of newborns from a public hospital located in the city of Rio das Ostras in the state of Rio de Janeiro, Brazil, participated in the study. Results: it was possible to verify that the different lexical analysis techniques available in IRAMUTEQ allowed to discriminate the semantic contents referring to the caregivers' claims
\end{abstract}

\footnotetext{
${ }^{\text {I }}$ Enfermeira. Doutora. Universidade Federal Fluminense, Rio das Ostras, RJ, Brasil. ferbezerra@gmail.com https://orcid.org/0000-0003-3894-3998 II Enfermeira. Universidade Federal Fluminense, Rio das Ostras, RJ, Brasil. torresandressa@hotmail.com https://orcid.org/0000-0001-7142-911X III Graduanda em Enfermagem. Universidade Federal Fluminense, Rio das Ostras, RJ, Brasil. brendalcampos@hotmail.com, https:/orcid.org/0000-0001-5832-3943 IV Enfermeira. Doutora. Universidade Federal Fluminense, Rio das Ostras, RJ, Brasil. alinecer2014@gmail.com https://orcid.org/0000-0002-8119-3945 V Enfermeira. Doutora. Universidade Federal Fluminense, Niterói, RJ, Brasil. lili.05@hotmail.com https://orcid.org/0000-0002-9125-1053
} VI Enfermeiro. Mestre. Universidade do Estado do Rio de Janeiro, Rio de Janeiro, RJ, Brasil. lcmoraesfranca@hotmail.com http://orcid.org/0000-0002-6370-115X 
about the care to be performed with the newborn at home. Conclusion: this computerized tool proved to be useful, adequate, and reliable, qualifying the textual analysis process.

Descriptors: Research; Nursing research; Qualitative research; Methodology; Software

Resumen: Objetivo: reportar la experiencia del uso del software Interface de R pour les Analyses Multidimensionnelles de Textes et de Questionnaires (IRAMUTEQ) como una herramienta computarizada para apoyar el análisis de datos textuales en una investigación cualitativa en salud. Método: se trata de un relato de experiencia que se llevó a cabo entre marzo y junio de 2019, con base en una investigación cualitativa desarrollada a través del Método Creativo-Sensible y utilizando IRAMUTEQ. Participaron recién nacidos y familiares de recién nacidos de un hospital público ubicado en la ciudad de Rio das Ostras en el estado de Rio de Janeiro, Brasil. Resultados: se pudo constatar que las diferentes técnicas de análisis léxico disponibles en IRAMUTEQ permitieron discriminar claramente los contenidos semánticos referidos a las afirmaciones de los cuidadores sobre el cuidado a realizar con el recién nacido en el domicilio. Conclusión: esta herramienta computarizada resultó ser útil, adecuada y confiable, calificando el proceso de análisis textual.

Descriptores: Investigación; Investigación en enfermería; Investigación cualitativa; Metodología; Programas informáticos

\section{Introdução}

No Brasil, nas últimas décadas, o desenvolvimento científico e tecnológico tem apresentado importantes avanços. ${ }^{1}$ Nesse cenário, profissionais de saúde, incluindo enfermeiros, estão conhecendo e incorporando novos métodos de pesquisa em suas investigações científicas. Entretanto, vivenciam desafios para garantir que os estudos propostos sejam relevantes e suficientemente rigorosos metodologicamente ${ }^{2}$ e se tornem publicados e, consequentemente, visíveis em periódicos com importantes fatores de impacto.

No campo da saúde, a pesquisa em enfermagem possui o papel de produzir e aprimorar conhecimentos, buscando a qualificação do cuidado e, assim, melhorias na qualidade de vida das pessoas. $^{3}$ Logo, a produção do conhecimento na área precisa contar com métodos de pesquisa robustos, que garantam a sistematização e a organicidade dos dados para uma adequada análise e interpretação dos achados, pelo pesquisador.

Dentre os tipos de pesquisa, a investigação qualitativa é uma abordagem metodológica frequentemente utilizada entre pesquisadores da saúde e da enfermagem, por apresentar uma visão idealista, subjetivista e interpretativa da realidade que é múltipla e subjetiva, possibilitando uma perspectiva mais compreensiva das relações humanas. Nessa abordagem, as experiências dos 
indivíduos e suas percepções são aspectos úteis e importantes para a pesquisa, ${ }^{4}$ o que leva à compreensão de fenômenos complexos no processo analítico.

O pesquisador que emprega o método qualitativo busca apreender a subjetividade dos indivíduos a partir de seu contexto. ${ }^{5}$ Entretanto, os dados brutos oriundos dessa abordagem são compostos por linguagens, por abarcarem pensamentos, crenças e/ou opiniões. Logo, a etapa de análise torna-se uma tarefa árdua, diante da subjetividade dos dados e do volume textual pouco estruturado, somados à busca premente de maior rigor metodológico. ${ }^{6-7}$

Uma alternativa viável é uso de softwares de análise, consolidados nas pesquisas quantitativas e que, desde a década de 1980, vêm crescendo dentre os investigadores qualitativos, com a denominação de Computer Aided Qualitative Data Analysis Software (CAQDAS). Entre as vantagens no processo analítico por esses softwares, destacam-se a organização e a separação de informações, o aumento na eficiência do processo e a facilidade de localização dos segmentos de texto. ${ }^{8}$

Dentre os CAQDAS, destaca-se o Interface de $R$ pourles Analyses Multidimensionnelles de Textes et de Questionnaires (IRAMUTEQ). Ele é um programa gratuito de processamento de dados textuais, criado pelo pesquisador francês Pierre Ratinaud, em seu idioma, mas que atualmente abarca diversos dicionários, incluindo o português, o que viabilizou o seu uso no Brasil, a partir de $2013^{7-9}$

O IRAMUTEQ visa apreender a estrutura e a organização dos enunciados, expondo as relações mais recorrentes entre os universos lexicais, admitindo que os vocábulos usados em contextos similares se correlacionam a um mesmo mundo lexical. ${ }^{7}$ Possibilita analisar entrevistas, documentos, redações, dinâmicas dentre outros, desde análises bem simples até multivariadas, oferecendo amplo leque de possibilidades de análises textuais, ${ }^{10}$ por vezes, pouco exploradas por pesquisadores em saúde.

Revisão de escopo que analisou 121 pesquisas qualitativas da área da saúde constatou que 
Utilização do software IRAMUTEQ em pesquisa de abordagem qualitativa: relato de ex.. $\quad$ |4

$21,5 \%$ dos estudos utilizaram softwares, sendo eles, Nvivo (18,2\%), Atlas.ti (1,7\%), Dedoose (0,8\%) e CQPweb software (0,8\%). ${ }^{5}$ Portanto, apesar da gratuidade do IRAMUTEQ, infere-se que os investigadores de pesquisas qualitativas em saúde ainda não estão familiarizados com esta ferramenta ou a desconhecem.

Verifica-se que é incipiente a produção de evidências com aplicação desse software na área da saúde, o que requer uma ampliação de estudos para o reconhecimento da ferramenta e que sirvam de referência para sua aplicabilidade, contribuindo para a formação metodológica de pesquisadores. Assim, objetivou-se relatar a experiência de utilização do IRAMUTEQ como ferramenta informatizada de apoio à análise de dados textuais em uma pesquisa qualitativa em saúde.

\section{Método}

Trata-se de um relato de experiência sobre a utilização do software IRAMUTEQ em uma pesquisa qualitativa, desenvolvida em um hospital público da cidade de Rio das Ostras no Estado do Rio de Janeiro, Brasil. Participaram 10 puérperas e nove familiares (cinco tias e quatro pais) de recém-nascidos, com idades iguais ou superiores a 18 anos.

Como técnica de produção de dados, foi empregada uma das dinâmicas de criatividade e sensibilidade do Método Criativo-Sensível (MCS), intitulada Dinâmica do Concreto. ${ }^{11}$ A sua utilização implicou no esclarecimento do cuidador mediante a execução de uma prática cuidativa, nesse estudo, referente aos cuidados pós-natais de recém-nascidos a serem desenvolvidos pelas famílias no domicílio, como higiene corporal e nutrição.

O MCS baseia-se no diálogo, na escuta e na solidariedade, privilegiando a participação ativa dos participantes na busca da construção coletiva do conhecimento, por meio de produções do tipo artísticas com base em uma Questão Geradora de Debate (QGD). O referido método combina estratégias típicas da pesquisa qualitativa tradicional, a saber, observação participante, entrevista 
coletiva e discussão grupal. ${ }^{12}$

Foram realizadas e totalizadas oito dinâmicas, sendo cada uma em cinco momentos conforme prevê o método. ${ }^{12}$ Cada encontro aconteceu em um ambiente reservado da instituição, entre março e junho de 2019, com duração média de 20 minutos. O papel dos participantes consistiu em demonstrar e falar sobre os cuidados que pretendiam realizar com o bebê em casa, separando os materiais a serem utilizados ou não, produzindo, assim, uma cena de cuidado fictícia, orientada pela seguinte QGD: Como você pretende realizar os cuidados com o seu recém-nascido em casa?

Foram disponibilizados materiais apropriados e inapropriados para os cuidados do recémnascido. Esses materiais incluíam chupeta, mamadeira, faixa umbilical, moeda, gaze, algodão, álcool 70\%, medicamento antisséptico contendo iodopovidona (povidine), cotonetes, fraldas, pomada contra assadura, lenço umedecido, shampoo, sabonete, perfume, talco, tesoura, dentre outros, além de um manequim estático de vinil com dimensões similares ao corpo de um bebê.

Posteriormente, no plano coletivo, as produções foram socializadas por meio da fala e da demonstração, de maneira espontânea, no grupo. Nesse processo, assuntos convergentes e divergentes geraram temas de debate, análise coletiva e discussão grupal. Por fim, ocorreu a síntese dos temas abordados e a validação das informações pelos próprios participantes. Esse tipo de dinâmica favoreceu a livre expressão de ideias, promovendo a geração de um volume léxico suficiente para o processamento de dados textuais no software selecionado.

Para um registro integral e preciso das falas, todas as Dinâmicas do Concreto sobre os cuidados pós-natais de recém-nascidos, foram gravadas em mídia digital, que depois de transcritas, geraram um único corpus textual, que se constituiu na fonte primária dos dados, submetido à análise lexicográfica, por meio do software IRAMUTEQ. Foram utilizados os cinco tipos de análise disponíveis: Estatísticas Textuais Clássicas; Nuvem de Palavras; Análise de Similitude; Classificação Hierárquica Descendente (CHD) e Análise Fatorial de Correspondência (AFC).

Para a correta utilização do software, são necessários alguns cuidados. Inicialmente, para 
Utilização do software IRAMUTEQ em pesquisa de abordagem qualitativa: relato de ex.. $\quad$ |6

concretizar a sua instalação, foram executados o download gratuito e a instalação no computador do software R, que possui linguagem e ambiente para análise estatística e produção de gráficos e, posteriormente, o do IRAMUTEQ, também de acesso livre. É essencial que essas etapas ocorram na ordem descrita, uma vez que o IRAMUTEQ se utiliza do software R no processamento das análises.

Além disso, outros procedimentos são necessários para o bom funcionamento do software. É comum que o IRAMUTEQ esteja disponibilizado em outro idioma, geralmente em francês, assim, foi efetuada a mudança de idioma, clicando em 'edição', depois em 'preferências' e em 'idioma da interface', com alteração para o português. Ao final, foi preciso encerrar o software para que ele salvasse as devidas modificações. Posteriormente, o programa foi reiniciado e a verificação de erros realizada, novamente clicando em 'edição', depois em 'preferências' e na sequência em 'verifique instalação de pacotes R', conforme instruções.

O presente estudo compõe um subprojeto da pesquisa primária, intitulada "Saberes e práticas de familiares acerca dos cuidados domiciliares com o recém-nascido de baixo risco”, aprovada pelo Comitê de Ética em Pesquisa, sob o nº 3.100.491, em 21 de dezembro de 2018. A pesquisa foi conduzida de acordo com as Resoluções 466/2012, 510/2016 e 580/2018 do Ministério da Saúde. Os dados foram produzidos após recrutamento e convite realizado pessoalmente pelas pesquisadoras, processo de consentimento e obtenção da assinatura do Termo de Consentimento Livre Esclarecido pelos participantes em ambiente reservado da instituição. Para o anonimato, utilizou-se um código alfanumérico relacionado aos 19 participantes do estudo [M - mães (1-10), P pais (1-4) e T- tias (1-5)], segundo a ordem de participação.

\section{Resultados}

Após as fases de instalação e ajustes necessários para o bom funcionamento do programa, já descritas, seguiu-se a análise dos dados textuais que ocorreu em três etapas: 1) preparação e codificação do corpus textual com a descrição do material empírico oriundo das dinâmicas 
desenvolvidas; 2) processamento dos dados textuais no software e 3) interpretação dos achados pelos pesquisadores (que não foram detalhados por não ser objeto de análise nesse relato de experiência).

Para a primeira etapa foram transcritas, na íntegra, as falas dos participantes, oriundas das oito dinâmicas de criatividade e sensibilidade com 10 puérperas e nove familiares de recémnascidos, com participação média de dois a três por dinâmica. Para a composição do corpus textual, as falas foram agrupadas por dinâmica, por causa do MCS que pressupõe um processo grupal, diferentemente das entrevistas individuais, na medida que o grupo passa a ser um espaço de múltiplas vozes dos partícipes da pesquisa, na enunciação das situações existenciais concretas, passíveis de socialização no espaço coletivo. Logo, os oito textos analisados no IRAMUTEQ foram oriundos das transcrições das oito dinâmicas, que conjuntamente originaram o corpus textual de análise, sendo ordenados em um arquivo de edição de texto e separados por uma linha de comando, a cada dinâmica, que necessariamente deve incluir quatro asteriscos, um espaço, um asterisco e uma identificação individual ou coletiva para o texto (**** Dinâmica_1 até *****Dinâmica_8).

Em seguida foi executada a revisão textual pelos pesquisadores, que excluíram do texto as intervenções e o material verbal que eles produziram, permanecendo as falas dos participantes e que respondiam à pergunta em foco. Foram corrigidos erros de digitação e pontuação, além da remoção de palavras, frases soltas e vícios de linguagem, como: “É...”, “Ihhh”, “Aî”, “Né”, “Tá”, “Tô”, “Ah”, “Oh”, “Hummm”, além de aspas ("), apóstrofo ('), hífen (-) e percentagem (\%).

Além disso, números foram mantidos em sua forma algarísmica e palavras compostas foram unificadas por meio do caractere underline “-”, em substituição aos espaços (exemplos: recém_nascido, lenço_umedecido, álcool_70, faixa_umbilical, sabão_de_coco, escova_de_dente, tesoura_com_ponta e tesoura_sem_ponta) para que o programa as contabilizasse como único termo.

Após o preparo do corpus textual, uma releitura atentiva foi realizada, para alcançar o maior aproveitamento possível, no processamento dos dados, do universo lexical que compunha o corpus de análise, visto que o IRAMUTEQ não possui uma função para verificação e correção de texto. 
Utilização do software IRAMUTEQ em pesquisa de abordagem qualitativa: relato de ex.. $\quad$ | 8

Posteriormente, esse arquivo único foi armazenado como documento de texto sem formatação (.txt) com a codificação de caracteres do tipo UTF-8 (Unicode Transformation Format 8 bit codeunits).

Para a segunda etapa, o primeiro passo foi 'abrir um corpus textual' no IRAMUTEQ e, posteriormente, na janela de definições, que se abre automaticamente, em 'definir caracteres' a opção “utf_8_sig - all languages” foi selecionada, bem como 'portuguese' no idioma e 'padrão' no dicionário. As outras configurações foram mantidas.

O primeiro tipo de análise é nomeada de “Estatística Textual Clássica”, que possibilita uma análise inicial do corpus, apresentando a relação entre a frequência e a quantidade de unidades léxicas no texto. Sem lematização (processo a ser selecionado ou não a cada tipo de análise), isto é, sem reduzir as palavras com base em suas raízes, o corpus textual gerado foi composto por oito textos, com uma totalidade de 8.124 ocorrências, sendo 1.160 vocábulos distintos, dos quais $51,2 \%$ de única ocorrência (hápax), com média de ocorrência por texto de 1.015,5 vocábulos.

Nessa análise é gerado o Diagrama de Zipf (Figura 1), uma forma visual de demonstrar o comportamento das palavras no corpus, ao ilustrar, no eixo vertical, a frequência de ocorrência das palavras ao longo do texto e, no eixo horizontal, o número de ordem/ranqueamento das palavras, com o valor 1 para a mais recorrente, 2 para a seguinte e, assim, sucessivamente. Ou seja, no eixo Y apresenta-se o logaritmo das frequências, ou quantas vezes uma palavra está presente no corpus textual, e no eixo X mostra-se a quantidade de palavras. Assim, foi possível observar uma curva decrescente no diagrama, no qual os pontos localizados no limite superior do gráfico, mais próximos ao Eixo Y, representam as palavras com uma alta frequência de repetição, porém menos recorrentes no material analisado, conforme indica o Eixo X. 


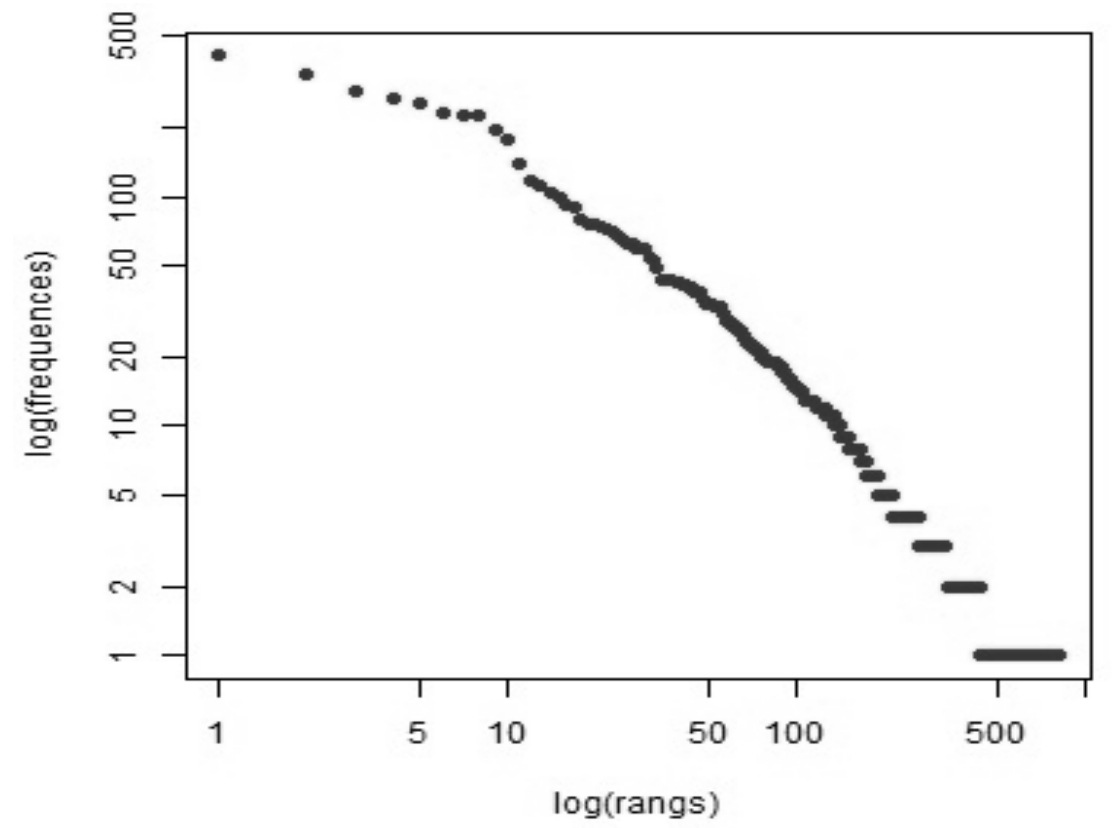

Figura 1 - Diagrama de Zipf com o comportamento das palavras no corpus textual sobre as pretensões de cuidadores quanto aos cuidados domiciliares ao recém-nascido. Rio das Ostras, RJ, Brasil, 2019.

Portanto, para o lado esquerdo da curva verifica-se que há poucas palavras que se repetem muitas vezes e do lado direito há muitas que se repetem poucas vezes. Por conseguinte, reforça-se que palavras com frequência 1 (hápax) foram recorrentes no texto ( $\mathrm{f}=594)$, como é visível ao final do Eixo X (linha horizontal). Por outro lado, apenas três formas ativas, após a lematização, apareceram mais de cem vezes nas dinâmicas transcritas, compondo o grupo das mais recorrentes, no topo do Eixo Y (linha vertical). Por ordem de ranqueamento essas formas ativas foram: não ( $\mathrm{f}=410)$, usar $(\mathrm{f}=224)$ e porque $(\mathrm{f}=105)$.

$\mathrm{Na}$ interpretação dos achados, verificou-se que o emprego desses termos se justifica pela dinâmica utilizada, na qual os participantes expuseram como realizariam os cuidados domiciliares com o bebê, separando os materiais que usariam ou não e explicando o porquê da escolha, como exemplifica o segmento de texto:

Não usaria faixa_umbilical. O pessoal colocava, mas hoje eu não uso, porque eu acho que o umbigo tapa e para curar, para secar, acho que vai ficar úmido. (P1)

Usaria a moeda e a faixa_umbilical. (M1) 
A análise por meio da "Nuvem de Palavras" projeta as palavras estruturadas em forma de nuvem, com tamanhos diferentes, sendo as maiores aquelas que detêm certa importância, por se repetirem mais vezes no corpus textual. Assim, com base no indicador de frequência, foi possível identificar as palavras de maior preponderância - as maiores e de forma mais central que as demais sem a utilização de um ponto de corte para a inclusão dos termos na nuvem (Figura 2). Verifica-se que os termos "não", "usar" e "porque” estão em destaque no conjunto das palavras, corroborando com a análise anterior.

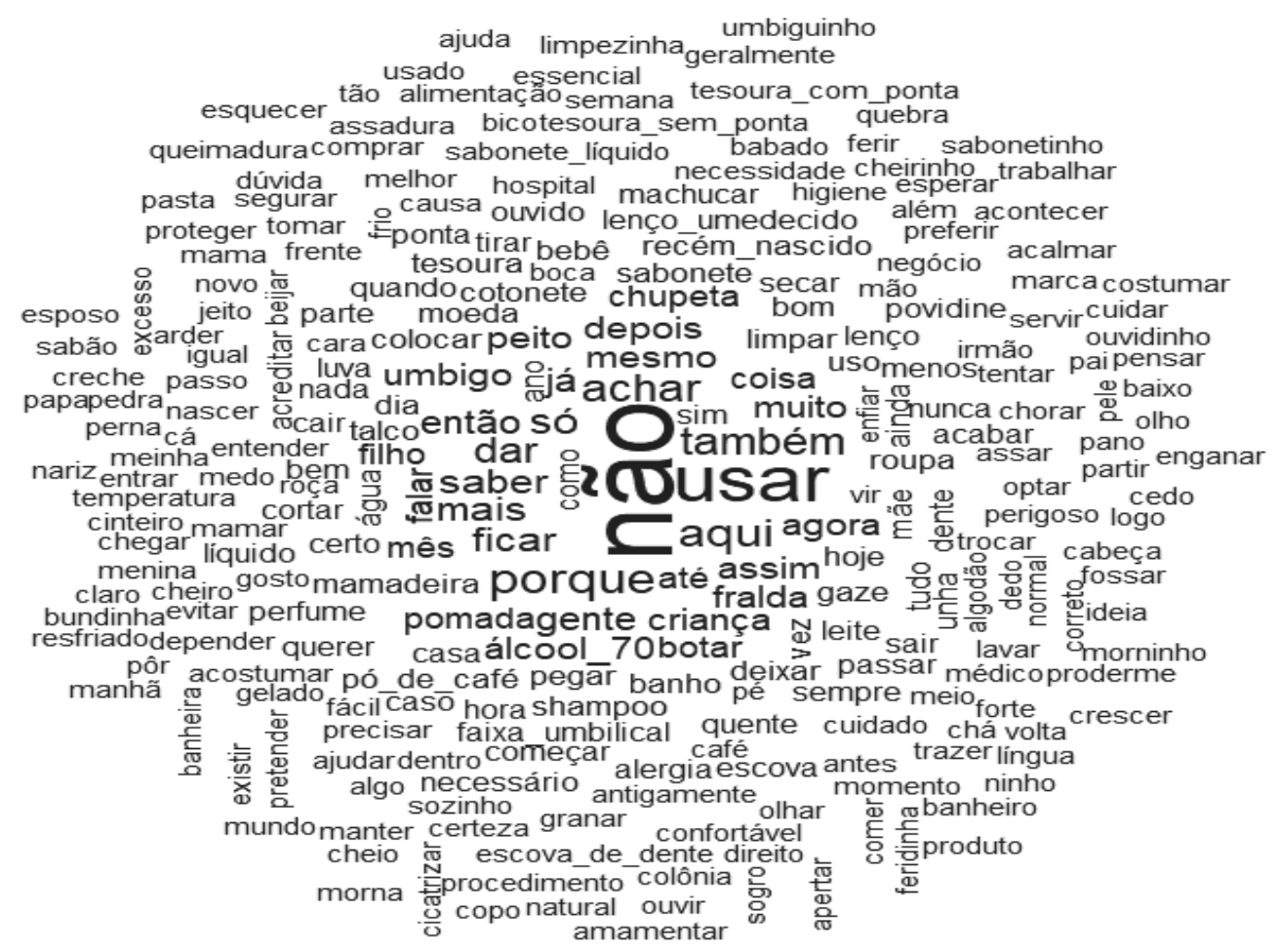

Figura 2 - Nuvem de palavras sobre as pretensões de cuidadores quanto aos cuidados domiciliares ao recém-nascido. Rio das Ostras, RJ, Brasil, 2019.

Foi possível averiguar que os materiais pronunciados pelas puérperas e familiares, com frequência de até dez repetições, para serem utilizados ou não nos cuidados aos recém-nascidos, foram: fralda ( $\mathrm{f}=43)$, álcool $70 \%(\mathrm{f}=38)$, pomada $(\mathrm{f}=33)$, chupeta $(\mathrm{f}=28)$, mamadeira $(\mathrm{f}=27)$, moeda $(\mathrm{f}=26)$, cotonete $(f=26)$, sabonete $(f=23)$, gaze $(f=21)$, shampoo $(f=21)$, roupa $(f=20)$, talco $(f=19)$, tesoura $(f=19)$, faixa umbilical ( $f=18)$, lenço umedecido $(f=16)$, perfume $(f=13)$, povidine (11) e escova ( $f=10)$. Ressaltase que é possível resgatar os segmentos de texto correspondentes aos termos no próprio software. 
Com a Análise de Similitude, baseada na teoria de grafos, foi viável identificar além das ocorrências das palavras, as indicações das conexidades (ligações) entre elas, o que auxilia na identificação da estrutura do conteúdo lexical (Figura 3). Destaca-se que para essa análise foram incluídas as palavras com recorrência de, no mínimo, 10 vezes. Observou-se que as três palavras que mais se destacaram (não, usar e porque), como já mencionado, assumiram posições de centralidade em três grupos de termos que geraram distintas ramificações, com realce novamente para o termo “não” por ser o mais central e com o maior número de conexões.

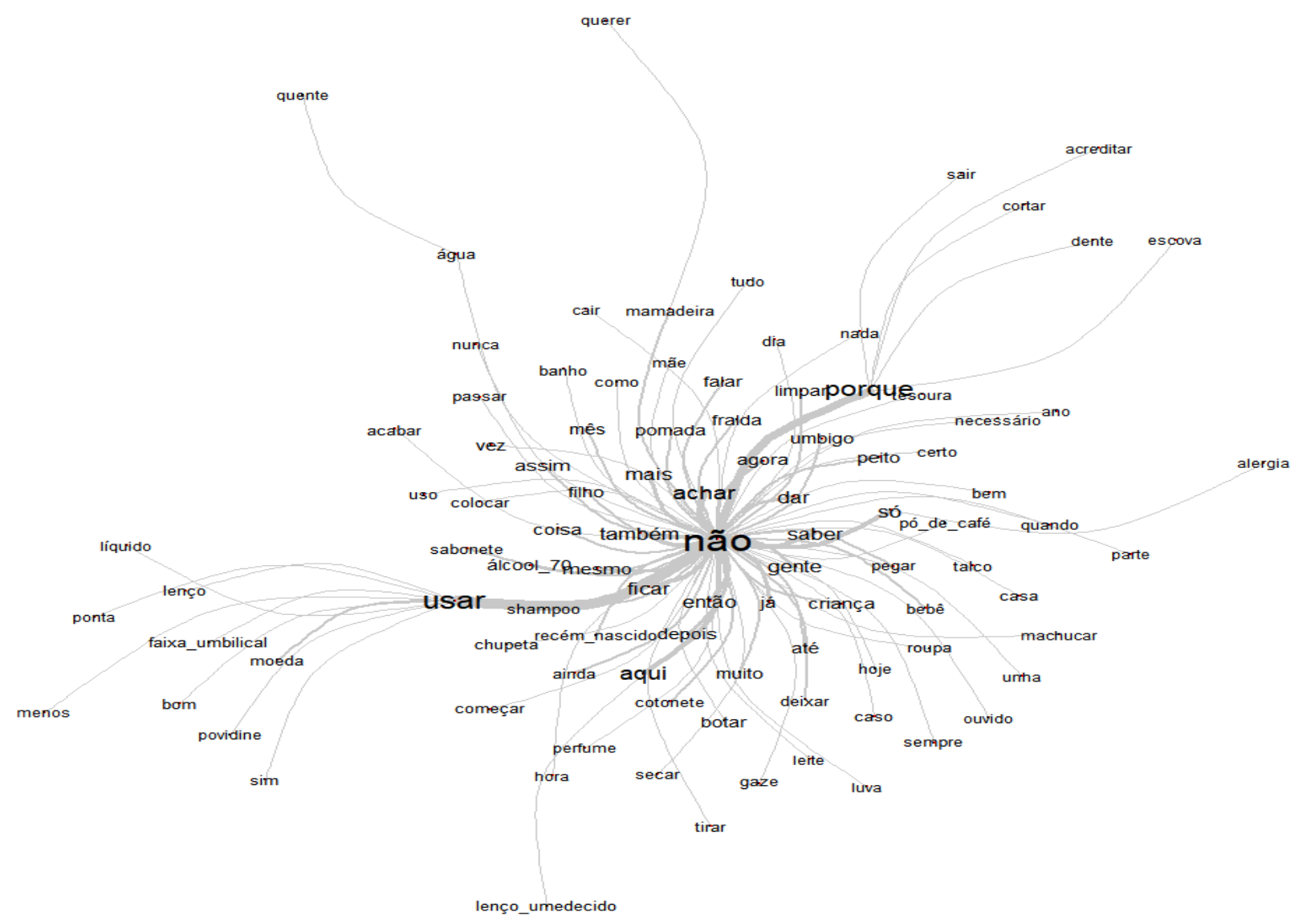

Figura 3 - Análise de Similitude com a ligação e as indicações de conexidade entre as palavras relacionadas às pretensões de cuidadores quanto aos cuidados domiciliares ao recém-nascido. Rio das Ostras, RJ, Brasil, 2019.

Na Análise de Similitude avança-se na compreensão das ramificações das palavras centrais para outros vocábulos que se conectam entre si, o que permitiu uma ampliação na interpretação dos dados. Assim, identificou-se o caminho pelo qual as palavras foram enunciadas e suas interligações dentre as quais, as mais fortes e recorrentes são representadas por linhas mais espessas. 
Portanto, pode-se inferir que como as falas dos participantes apresentaram referências que fazem parte do processo de cuidar de recém-nascidos no domicílio, houve a elucidação minuciosa 'do que usar', 'do como usar' e 'do que não usar' no cuidado de seus bebês, abarcando, inclusive, os porquês das escolhas indicadas, como revelam os segmentos de texto a seguir:

Fralda, pomada também, eu uso mesmo que não esteja com nada, passo para prevenir, a cada troca de fralda. Mamadeira ainda não, porque pretendo deixar ele só no peito até uns 6 meses. (P10)

Usaria a chupeta, vou passar na farmácia para comprar, porque aqui não pode. (P9)

Usaria essa tesoura_sem_ponta, essa aqui tem ponta, pode machucar(T1).

Outro método utilizado para análise foi a CHD, no qual os segmentos de texto são agrupados em função de seus respectivos vocabulários e o conjunto deles é repartido em função da presença ou da ausência das formas lematizadas constituindo, assim, distintos mundos lexicais. Esse método estabelece uma classificação estável e definitiva, por meio de classes de segmentos de texto que, ao mesmo tempo, apresentam vocabulário semelhante entre si, de acordo com parâmetros léxicos em comum e vocabulário diferente dos segmentos de texto das outras classes, mediante cálculos de distanciamentos e proximidades a partir de testes de qui-quadrado $\left(\chi^{2}\right)$.

Assim, o software organiza a análise dos dados em diferentes layouts de dendrogramas na CHD, que ilustram as relações entre as classes. Por esta análise, foram encontrados 243 segmentos de texto, com classificação de 203 deles, ou seja, houve um aproveitamento de 83,54\%. A lematização resultou em 803 lemas e, dentre as formas ativas, 705 eram analisáveis e 262 apresentaram frequência $\geq$ três. Na CHD foram geradas seis classes de segmentos de textos distintas (Figura 4) que demonstra os conteúdos semânticos de cada classe. 


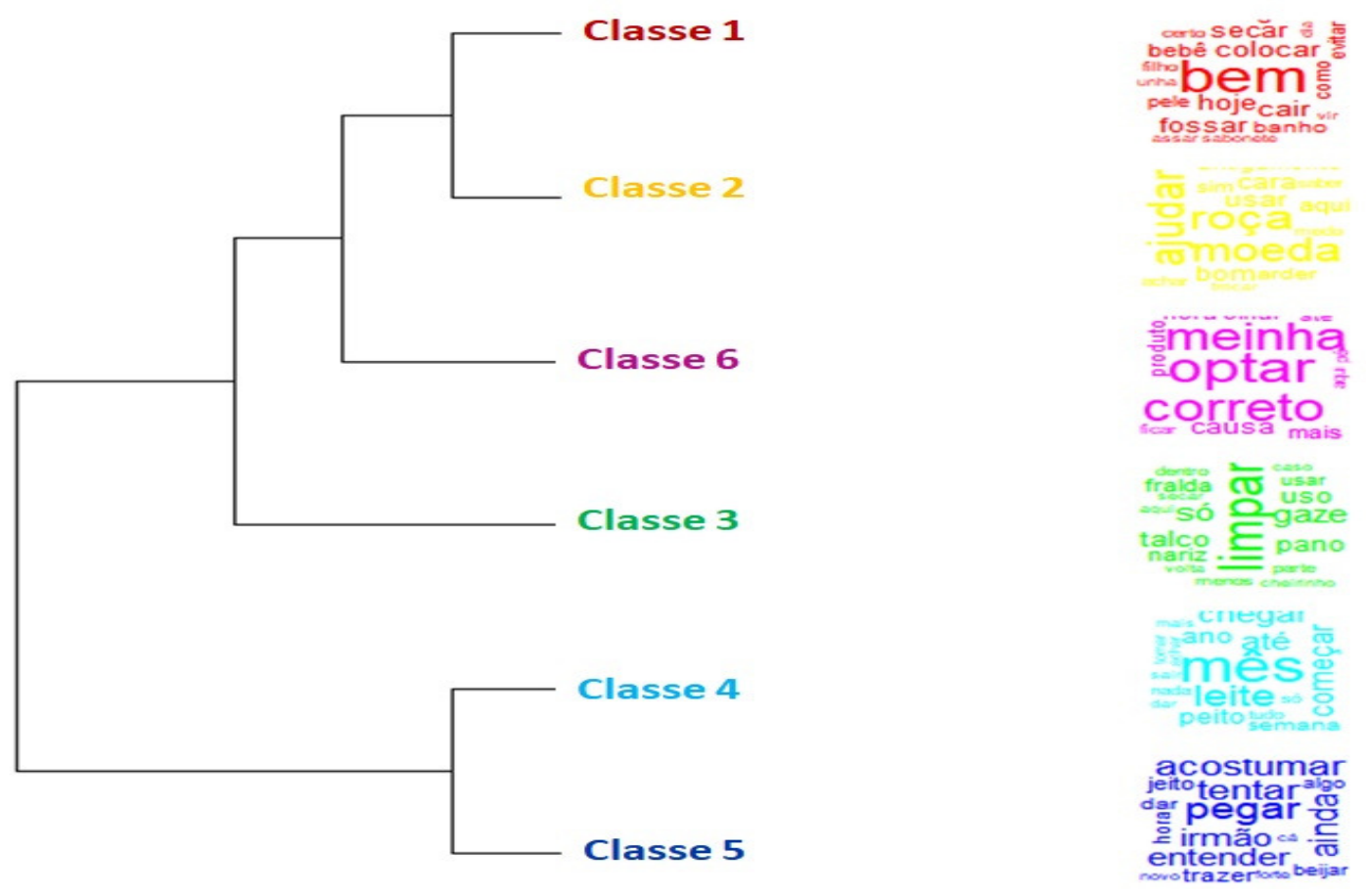

Figura 4 - Dendrograma na Classificação Hierárquica Descendente dos segmentos de texto relacionados às pretensões de cuidadores quanto aos cuidados domiciliares ao recém-nascido. Rio das Ostras, RJ, Brasil, 2019.

A análise apresentou estabilidade e, consequentemente, as seis classes foram estáveis, ou seja, foram compostas por segmentos de texto com vocábulos semelhantes. No dendrograma, o corpus textual foi particionado em dois blocos (subcorpus) independentes. O primeiro foi composto pela classe $3(21,2 \%)$, por uma segunda subdivisão incluindo a classe $6(17,2 \%)$ e por outra subdivisão com as classes $2(12,3 \%)$ e $1(13,8 \%)$, as quais possuem conteúdos semânticos mais próximos mas, ainda, com certa diferenciação. O outro bloco foi formado pelas classes $4(15,8 \%)$ e 5 (19,7\%), sendo as mais isoladas das demais, o que demonstra maior proximidade e homogeneidade entre as duas.

$\mathrm{Na}$ interpretação dos dados, a partir da CHD, foram resgatadas as formas ativas de cada classe de segmentos de texto, que incluiu substantivos, adjetivos, advérbios, verbos e formas não reconhecidas, com destaque para as que obtiveram no teste qui-quadrado $\left(\chi^{2}\right)$ um valor $\geq 3,84$, logo, $\mathrm{p}<0,05$, que revela a força de associação das palavras em uma respectiva classe. Quanto menor for o $\chi^{2}$, menos os termos estão relacionados à classe; por outro lado, quanto menor for o p-valor, maior essa relação, o que colabora para a validação e confiabilidade dos achados. Assim, os vocábulos cujos valores de $\mathrm{p}$ foram menores que 0,0001 são extremamente significantes em cada classe, ao 
denotarem com mais de $99,99 \%$ de certeza que suas alocações na classe não foram ao acaso.

Nessa diretiva, após o processamento dos dados pelo software, procedeu-se a leitura das palavras significativas nas classes e de suas inserções nos segmentos de textos. Portanto, a partir dos conteúdos semânticos, as classes foram denominadas: Classe 1: Cuidados com o banho do recém-nascido; Classe 2: Cuidados com o coto umbilical; Classe 3: Materiais utilizados na higiene do recém-nascido; Classe 4: Nutrição do recém-nascido; Classe 5: Uso de bicos artificiais; Classe 6: Poder de decisão no cuidado ao recém-nascido.

Reforça-se que esta nomeação, realizada pelos autores, considerou o universo semântico de cada classe. Ou seja, a partir das principais palavras associadas, da leitura exaustiva das mesmas e do contexto semântico em que se encontravam inseridas, o sentido das classes foi extraído, levando à sua nomeação. A interpretação do conjunto dessas repartições permitiu alcançar a compreensão de como os participantes pretendiam realizar os cuidados domiciliares com o recém-nascido.

O programa oferece outra forma de apresentação dos resultados, por meio da AFC que representa, num plano cartesiano, os vocábulos e posicionamento das classes, a partir das frequências e valores de correlação $\left(\chi^{2}\right)$ de cada palavra (Figura 5), favorecendo a visualização do vocabulário típico de cada classe em distintos mundos lexicais ou contextos semânticos. 


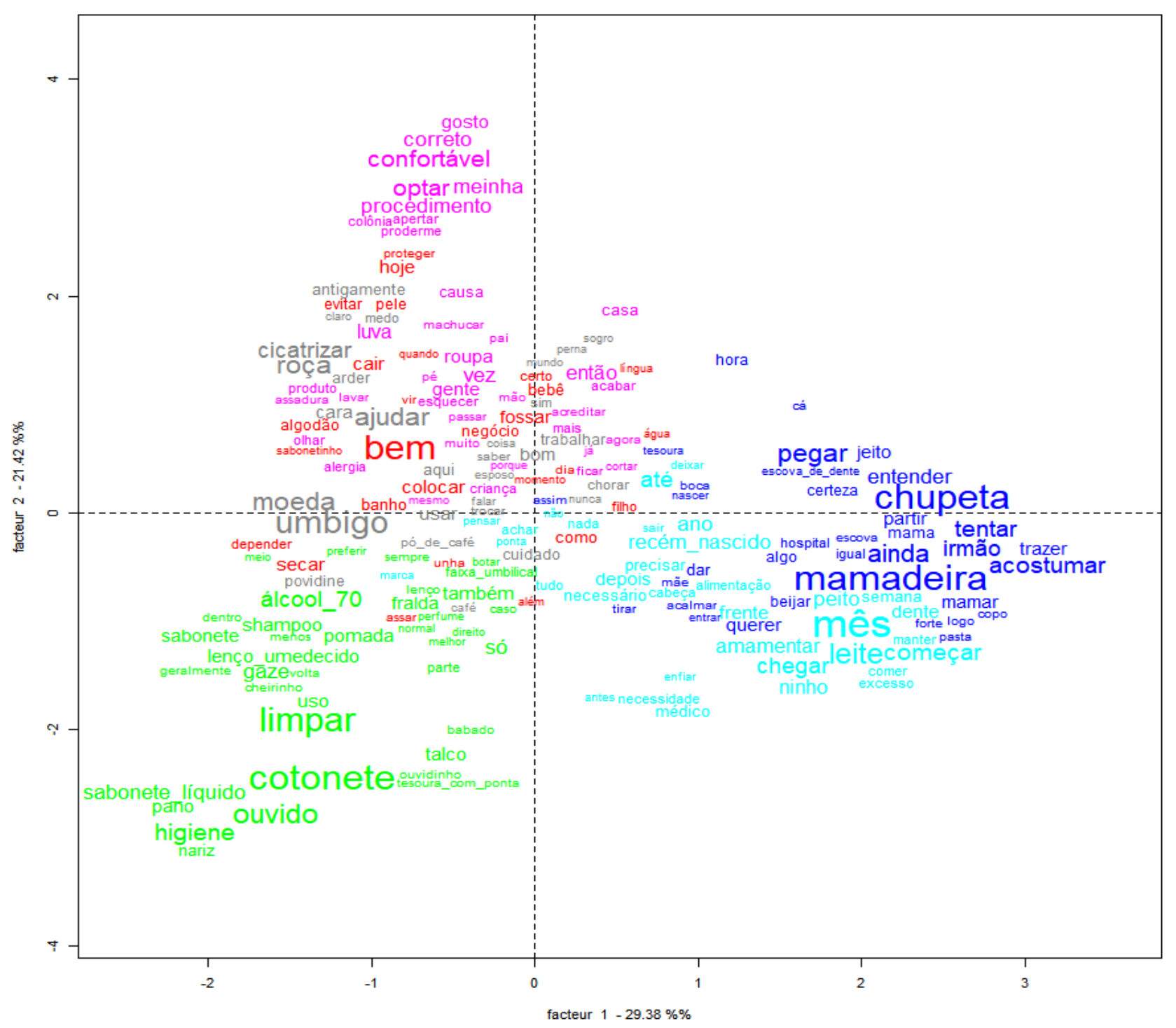

Figura 5 - Análise Fatorial por Correspondência (AFC) dos segmentos de texto relacionados às pretensões de cuidadores quanto aos cuidados domiciliares ao recém-nascido. Rio das Ostras, RJ, Brasil, 2019.

A partir da AFC, verifica-se que, em geral, as palavras mais importantes, com $\chi^{2}$ maiores, logo, de tamanhos maiores na imagem, de todas as classes apresentam-se num segmento mais centralizado, enquanto as outras expandem-se para a periferia. Ademais, poucas palavras de cada classe dispersam-se aos outros quadrantes. Chama atenção que a classe 3 (quadrante inferior esquerdo), na cor verde, é a mais isolada no plano cartesiano, sem avançar para nenhum outro quadrante; portanto, menos associada às outras classes e com um mundo lexical muito particular. $\mathrm{Na}$ CHD, esta foi a primeira classe constituída, abrangendo uma lista de materiais específicos relacionados à higiene do recém-nascido. 
Também foi possível confirmar que as classes 1, 2 e 6, aqui apresentadas nas cores vermelha, cinza e rosa (quadrante superior esquerdo), aparecem associadas entre si. Isto ocorre na medida que os cuidados com banho do recém-nascido e com o coto umbilical estão interligados nas falas das puérperas e familiares, assim como as questões referentes ao poder de decisão dos participantes, logo, as palavras das três classes mesclam-se no quadrante.

$\mathrm{Na}$ análise interpretativa, verificou-se que, principalmente, os familiares que possuíam conhecimentos prévios em cuidados com recém-nascidos, optavam pelo modo que acreditavam ser correto na execução de uma prática cuidativa, demonstrando autonomia em suas escolhas.

Por fim, as classes 4 e 5, em azul claro e azul escuro (quadrante inferior direito), estão correlacionadas no plano fatorial, por terem vocabulários mais próximos referentes à nutrição do recém-nascido. Elas incluem a amamentação e o uso ou não de bicos artificiais, como chupetas e mamadeiras, justificando sua proximidade.

Cumpre salientar que a utilização do IRAMUTEQ nessa pesquisa qualitativa foi de grande valia para os pesquisadores envolvidos, pois permitiu o aprimoramento de seus conhecimentos no manejo de um software analítico. Ademais, reforça-se que sua aplicabilidade favorece a operacionalização dos dados, principalmente, quanto à organização e separação de um grande volume textual de forma rápida e singular, quando o corpus é devidamente e antecipadamente preparado. Logo, facilita-se, inclusive, a interpretação dos achados de maneira objetiva, na medida em que os segmentos de texto são facilmente recuperados. Outra vantagem percebida foi a possibilidade de todos os pesquisadores terem acesso ao software, por ser gratuito e com dicionário em português, o que possibilitou encontros para treinamentos e discussões na etapa de processamento dos dados textuais, com a participação efetiva de todos os envolvidos na pesquisa, que aprenderam a manejar essa ferramenta. 


\section{Discussão}

O uso do IRAMUTEQ foi projetado para grandes volumes textuais. Desde que o grupo seja homogêneo, em geral, recomenda-se entre 20 e 30 textos. $^{7}$ No presente estudo, embora com 19 familiares, o corpus textual foi classificado como aproveitável e adequado para a realização deste tipo de análise, pois alcançou percentagem superior da recomendada pela literatura de $75 \% .^{7-8}$

O nível de aproveitamento de $83,54 \%$ pode ser justificado pela utilização do MCS, que por empregar a arte como ferramenta para a expressão livre de situações existenciais, criou um espaço de discussão e reflexão mediado pelo diálogo. Os indivíduos problematizaram suas práticas vivenciais e existenciais sobre o tema em tela de forma livre e menos mecanizada, ${ }^{12}$ gerando dados suficientes para o processamento no IRAMUTEQ.

O número de participantes foi delimitado pela saturação dos dados, identificada durante a organização do material empírico oriundo das dinâmicas, mediante a redundância informacional das falas dos participantes. ${ }^{13}$ Considerou-se saturada a coleta quando nenhum novo elemento foi encontrado, passando a existir recorrência de ideias sobre a temática nas dinâmicas e sem o acréscimo de novas informações, ${ }^{14}$ o que foi confirmado pelo nível de aproveitamento.

As diferentes técnicas de análise lexical permitiram discriminar os conteúdos semânticos referentes às pretensões das puérperas e dos familiares sobre os cuidados a serem desenvolvidos com o recém-nascido em casa, incluindo distintos materiais e prática, além dos motivos para suas escolhas. Logo, verificou-se que o uso desse software garantiu o rigor estatístico diante da necessidade de lidar com respostas textuais extensas; do aprimoramento das análises, especialmente, pela integração entre os domínios quantitativos e qualitativos no processo analítico e de maior objetividade na interpretação em pesquisas de abordagem qualitativa. ${ }^{15}$ Além disso, constatou-se que sua interface é simples e de fácil compreensão, conforme indica a literatura. ${ }^{10}$

Pelo diagrama de Zipf, várias palavras foram pouco recorrentes e raras palavras repetiram-se muito, sendo que as três mais citadas se apresentaram relevantes em todo o processo analítico, 
permanecendo em posições centrais e de destaque na Nuvem de Palavras e na Análise de Similitude. A primeira, mesmo sendo uma análise lexical mais simples, foi útil e interessante, pois, ao possibilitar a rápida identificação das palavras-chave de um corpus, ${ }^{7,10}$ favoreceu, inclusive, a identificação da lista de materiais a serem utilizados no cuidado do bebê.

A Análise de Similitude, baseada em um modelo matemático para estudo das relações entre objetos, permitiu identificar as co-ocorrências entre as palavras, o que indicou seus vínculos, auxiliando na identificação da estrutura do corpus ${ }^{16}$ Essa análise de semelhanças contribuiu para a visualização de como os participantes relacionaram as palavras para descrever o processo de cuidar de recém-nascidos no domicílio, incluindo o que usar ou não e os porquês das opções.

A CHD tem sido a forma de análise mais utilizada nas pesquisas que usam o IRAMUTEQ. ${ }^{7}$ Nela foi gerado um dendrograma com seis classes, contendo as diferentes palavras que obtiveram maior índice de associação em cada uma das classes, mediante a aplicação de um teste estatístico amplamente conhecido em estudos de abordagem quantitativa, o qui-quadrado de Pearson, que quanto maior, mais provável é a hipótese de dependência entre a palavra ativa e a classe. Essa análise indicou um elevado poder de distinção do software em relação à representatividade dos conjuntos de ideias dos participantes. ${ }^{15}$

Esse recurso possibilitou, com base no corpus, a recuperação dos segmentos de textos e a associação das palavras, que permitiu o agrupamento dos vocábulos estatisticamente significantes, com posterior análise interpretativa pelos pesquisadores, favorecendo uma análise mais precisa e assertiva dos dados, ${ }^{8,17}$ ao distinguir classes que são, ao mesmo tempo, suficientemente distintas das demais e suficientemente semelhantes entre si, no que tange ao vocabulário.

Com a AFC foi possível averiguar uma clara diferença na distribuição das palavras ao longo do cruzamento dos eixos X e Y no plano fatorial, contudo, em consonância com a repartição e a interligação entre as classes fornecidas pela CHD. Tal interface favorece a avaliação minuciosa das ligações entre os perfis das respostas individuais, expressando convergências e oposições e 
mostrando, graficamente, os vínculos existentes entre os diferentes mundos lexicais. ${ }^{18}$ Nessa diretiva, os conteúdos semânticos relacionados à higiene e à nutrição do bebê ficaram em planos nitidamente distintos, o que se apresenta como um importante diferencial no uso do IRAMUTEQ.

Diante do exposto, reforça-se a importância do conhecimento das distintas alternativas de processamento de texto oferecidas pelo software para utilizá-lo corretamente. Logo, enfatiza-se a relevância do pesquisador em todas as fases da pesquisa, com o devido aporte teórico para a preparação do corpus textual. ${ }^{19}$ Além disso, a análise de dados, a partir do IRAMUTEQ, não pode perder de vista o problema de pesquisa, que precisa nortear os processos de coleta, tratamento, análise e interpretação de dados, bem como os processos inferenciais devem estar apoiados nos marcos teóricos do estudo e nas reflexões críticas sobre a validade e o significado dos achados. ${ }^{20}$

Algumas limitações na aplicação do software precisam ser ressaltadas. Por se tratar de uma ferramenta de auxílio na análise, a interpretação do que pode estar por trás das semelhanças e diferenças lexicais é uma tarefa que ultrapassa os limites do software, portanto, depende dos marcos conceituais e/ou construtos teóricos adotados pelos pesquisadores. Além disso, erros de codificação nas linhas de comando, de português e palavras coloquiais, não são processados pelo software, cabendo ao pesquisador identificar tais erros e corrigi-los, sem alterar o sentido das falas. Logo, requer atenção na transformação do conteúdo transcrito em corpus que será analisado e, assim, obter um bom aproveitamento textual.

\section{Conclusão}

O estudo relatou a experiência de uso do software IRAMUTEQ como uma ferramenta informatizada de apoio à análise de dados textuais resultantes na pesquisa qualitativa em saúde. A utilização do software foi útil, adequada, precisa e fidedigna, qualificando o processo de análise e, por conseguinte, os resultados da pesquisa, possibilitando sua interpretação com o devido rigor científico. Logo, o uso desta ferramenta potencializou a análise dos dados oriundos das dinâmicas 
de criatividade e sensibilidade.

A escassez de pesquisas que divulgam a utilização desse software, no Brasil, com a descrição dos diferentes tipos de análise foi um desafio encontrado, o que por outro lado, reforçou a importância desse estudo que descreveu o processo analítico contemplando todas as interfaces disponíveis. Portanto, destaca-se a necessidade de novos estudos que apliquem o IRAMUTEQ, com o propósito de ampliar a compreensão e a utilização dessa ferramenta nas pesquisas de abordagem qualitativa na área da saúde e da enfermagem.

\section{Referências}

1. Souza DL, Zambalde AL, Mesquita DL, Souza TA, Silva NLC. A perspectiva dos pesquisadores sobre os desafios da pesquisa no Brasil. Educ Pesqui. 2020;46:e221628. doi: 10.1590/s1678-4634202046221628

2. Thorne S. Making our nursing research matter. Rev Bras Enferm. 2016;69(5):813-4. doi:10.1590/0034-71672015-0156

3. Erdmann AL, Peiter CC, Lanzoni GMM. Brazilian research groups in nursing: comparison of 2006 and 2016 profiles. Rev Gaúcha Enferm. 2017;38(2):e69051. doi: 10.1590/1983-1447.2017.02.69051

4. Patias ND, Hohendorff JV. Critérios de qualidade para artigos de pesquisa qualitativa. Psicol Estud. 2019; 24:e43536. doi: 10.4025/psicolestud.v24i0.43536

5. Salvador PTCO, Alves KYA, Rodrigues CCFL, Oliveira LV. Online data collection strategies used in qualitative research of the health field: a scoping review. Rev Gaúcha Enferm. 2020;41:e20190297. doi: 10.1590/1983-1447.2020.20190297

6. Nunes JV, Woloszyn M, Gonçalves BS, Pinto MDS. A pesquisa qualitativa apoiada por softwares de análise de dados: uma investigação a partir de exemplos. Rev Front. 2017;19(2):233-44. doi: 10.4013/fem.2017.192.08

7. Salvador PTCO, Gomes ATL, Rodrigues CCFM, Chiavone FBT, Alves KYA, Bezerril MS, et al. Uso do software IRAMUTEQ nas pesquisas brasileiras da área da saúde: uma scoping review. Rev Bras Promoç Saúde. 2018;31:1-9. doi: 10.5020/18061230.2018.8645

8. Souza MAR, Wall ML, Thuler ACMC, Lowen IMV, Peres AM. The use of IRAMUTEQ software for data analysis in qualitative research. Rev Esc Enferm USP. 2018;52:e03353. doi: 10.1590/S1980-220X2017015003353

9. Kami MTM, Larocca LM, Chaves MMN, Lowen IMV, Souza VMP, Goto DYN. Working in the street clinic: use of IRAMUTEQ software on the support of qualitative research. Esc Anna Nery. 2016;20(3):e20160069. doi: 10.5935/1414-8145.20160069

10. Camargo B, Justo AM. IRAMUTEQ: um software gratuito para análise de dados textuais. Temas Psicol. 2013; 21(2):513-18. doi: 10.9788/TP2013.2-16 
11. Gomes AMT, Cabral IE. Ocultamento e silenciamento familiares no cuidado à criança em terapia antiretroviral. Rev Bras Enferm. 2010;63(5):719-26. doi: 10.1590/S0034-71672010000500005

12. Soratto J, Pires DEP, Cabral IE, Lazzari DD, Witt RR, Sipriano CAS. A maneira criativa e sensível de pesquisar. Rev Bras Enferm. 2014;67(6):994-9. doi: 10.1590/0034-7167.2014670619

13. Saunders B, Sim J, Kingstone T, Baker S, Waterfield J, Bartlam B, et al. Saturation in qualitative research: exploring its conceptualization and operationalization. Qual Quant. 2018;52(4):1893-907. doi: 10.1007/s11135017-0574-8

14. Nascimento LCN, Souza TV, Oliveira ICS, Moraes JRMM, Aguiar RCB, Silva LF. Theoretical saturation in qualitative research: an experience report in interview with schoolchildren. Rev Bras Enferm. 2018;71(1):228-33. doi: 10.1590/0034-7167-2016-0616

15. Carvalho TS, Mota DM, Saab F. Utilização do software IRaMuTeQ na análise de contribuições da sociedade em processo regulatório conduzido pela Agência Nacional de Vigilância Sanitária. Vigil Sanit Debate. 2020;8(1):10-21. doi: 10.22239/2317-269x.01429

16. Mendes FRP, Zangão MOB, Gemito MLGP, Serra ICC. Social Representations of nursing students about hospital assistance and primary health care. Rev Bras Enferm. 2016;69(2):321-8. doi: 10.1590/00347167.2016690218i

17. Silva AMB, Enumo SRF. Description and analysis of psychological intervention with dancers by the Software IRAMUTEQ. Temas Psicol. 2017;25(2):577-93. doi: 10.9788/TP2017.2-11Pt

18. Trigueiro DRSG, Almeida AS, Monroe AA, Costa GO, Bezerra VP, Nogueira JA. AIDS and jail: social representations of women in freedom deprivation situations. Rev Esc Enferm USP. 2016; 50(4):554-61. doi: 10.1590/S0080-623420160000500003

19. Acauan LV, Abrantes CV, Stipp MAC, Trotte LAC, Paes GO, Queiroz ABA. Utilização do software Iramuteq ${ }^{\circledR}$ para análise de dados qualitativos na Enfermagem: um ensaio reflexivo. REME Rev Min Enferm. 2020;24:e1326. doi: 10.5935/1415-2762.20200063

20. Sousa YSO, Gondim SMG, Carias IA, Batista JS, Machado KCM. O uso do software Iramuteq na análise de dados de entrevistas. Pesqui Prát Psicossociais [Internet]. 2020 [acesso em 2020 nov 29];15(2):1-19. Disponível em: http://pepsic.bvsalud.org/scielo.php?script=sci_arttext\&pid=S1809$89082020000200015 \& \operatorname{lng}=\mathrm{pt} \& \mathrm{t} \operatorname{lng}=\mathrm{pt}$

Editora Científica Chefe: Cristiane Cardoso de Paula

Editora associada: Graciela Dutra Sehnem

Fomento/Agradecimento: Ao Conselho Nacional de Desenvolvimento Científico e Tecnológico (CNPq) pela Bolsa de Iniciação Científica. 


\section{Autor correspondente}

Fernanda Garcia Bezerra Góes

E-mail: ferbezerra@gmail.com

Endereço: Rua Recife, Lotes, 1-7 - Jardim Bela Vista, Rio das Ostras/RJ. CEP: 28895-532

\section{Contribuições de Autoria}

\section{1 - Fernanda Garcia Bezerra Góes}

Contribuições: Colaborou com a concepção e desenho do estudo, na análise e interpretação dos dados e na revisão final com participação crítica e intelectual no manuscrito.

\section{2 - Andressa Silva Torres dos Santos}

Colaborou com a concepção e desenho do estudo, na análise e interpretação dos dados e na revisão final com participação crítica e intelectual no manuscrito.

\section{3 - Brenda Lucas Campos}

Colaborou com a concepção e desenho do estudo, na análise e interpretação dos dados e na revisão final com participação crítica e intelectual no manuscrito.

\section{4 - Aline Cerqueira Santos Santana da Silva}

Colaborou na análise e interpretação dos dados e na revisão final com participação crítica e intelectual no manuscrito.

\section{5 - Liliane Faria da Silva}

Colaborou na análise e interpretação dos dados e na revisão final com participação crítica e intelectual no manuscrito.

\section{6 - Luiz Carlos Moraes França}

Colaborou na análise e interpretação dos dados e na revisão final com participação crítica e intelectual no manuscrito.

\section{Como citar este artigo}

Góes FGB, Santos AST, Campos BL, Silva ACSS, Silva LF, França LCM. Use of the IRAMUTEQ software in research with a qualitative approach: experience report. Rev. Enferm. UFSM. 2021 [Access on: Anos Mês Dia]; vol.11 e63: 1-22. DOI: https://doi.org/10.5902/2179769264425 